\title{
Actividad física y su relación con el rendimiento académico
}

\section{Physical Activity and Its Relationship with Academic Performance}

Atividade física e sua relação com o desempenho acadêmico

Francisco De Jesús Ávila Manríquez

Universidad Autónoma de Querétaro, México francisco219108@gmail.com

https://orcid.org/0000-0002-8256-2570

Julio César Méndez Ávila

Universidad Autónoma de Querétaro, México

julio_uaq@hotmail.com

https://orcid.org/0000-0002-4059-2288

José Miguel Silva Llaca

Universidad Autónoma de Querétaro, México msilva_uaq@hotmail.com https://orcid.org/0000-0002-1527-0033

Oscar Ángel Gómez Terán

Universidad Autónoma de Querétaro, México oagomter@gmail.com https://orcid.org/0000-0001-6637-5217 


\section{Resumen}

El objetivo de esta investigación fue determinar la eficacia que tiene un programa de actividad física sobre el rendimiento escolar de estudiantes de una escuela primaria pública del municipio de Querétaro, México. La metodología que se utilizó fue cuantitativa, de alcance descriptivo y correlacional. Se aplicó un diseño cuasiexperimental de pretest, postest y grupo control con una intervención durante nueve meses a 73 estudiantes. Los instrumentos de recolección de datos fueron un cuestionario, que obtuvo un coeficiente de fiabilidad de alfa de Cronbach de 0.796, una encuesta sobre el nivel socioeconómico, una batería de coordinación motriz (KTK) y un instrumento para medir el rendimiento académico (TERA). Para los análisis estadísticos se utilizaron las técnicas estadísticas de ji al cuadrado y la prueba $t$ de Student. Dentro de los resultados se encontró una asociación significativa entre la actividad física y el rendimiento académico; también se halló una correlación en la actividad física y el desarrollo motriz en los estudiantes. Sin embargo, no se reveló asociación alguna entre el nivel socioeconómico y las variables de rendimiento académico y actividad física. Se concluye que no existen hábitos sobre la actividad física, que las horas destinadas a la práctica de la actividad física en las escuelas son insuficientes y que el rendimiento académico en las instituciones de nivel básico presenta atrasos educativos que deben ser atendidos de manera integral.

Palabras claves: actividad física, nivel socioeconómico, rendimiento académico.

\section{Abstract}

The objective of this research was to determine the effectiveness of a physical activity program on the school performance of students from a public primary school in the municipality of Querétaro, Mexico. The methodology used was quantitative, descriptive, and correlational. A quasi-experimental design was applied, with a pretest, posttest and control group, and with an intervention for nine months to 73 students. The data collection instruments were a questionnaire, which obtained a Cronbach's alpha reliability coefficient of 0.796 , a survey on socioeconomic level, a motor coordination battery (KTK) and an instrument to measure academic performance (TERA). Within the results, a significant association was found between physical activity and academic performance; a correlation was also found in physical activity and motor development in students. However, no association was revealed between socioeconomic level and the variables academic performance and physical activity. It is concluded that there are no habits about 
physical activity, that the hours allocated to the practice of physical activity in schools are insufficient and that academic performance in basic-level institutions presents educational delays that must be addressed in a comprehensive manner.

Keywords: physical activity, socioeconomic level, academic performance.

\section{Resumo}

O objetivo desta pesquisa foi determinar a eficácia de um programa de atividade física no desempenho escolar de alunos de uma escola pública de ensino fundamental do município de Querétaro, no México. A metodologia utilizada foi quantitativa, descritiva e correlacional em escopo. Um desenho quase-experimental de pré-teste, pós-teste e grupo de controle com uma intervenção de nove meses foi aplicado a 73 alunos. Os instrumentos de coleta de dados foram um questionário, que obteve coeficiente de confiabilidade alfa de Cronbach de 0,796, levantamento de nível socioeconômico, bateria de coordenação motora (KTK) e instrumento de medida de desempenho acadêmico (TERA). Técnicas estatísticas de qui-quadrado e teste $\mathrm{t}$ de Student foram usados para análises estatísticas. Dentre os resultados, foi encontrada associação significativa entre atividade física e desempenho acadêmico; uma correlação também foi encontrada na atividade física e desenvolvimento motor em alunos. No entanto, não foi revelada associação entre nível socioeconômico e as variáveis de desempenho acadêmico e atividade física. Conclui-se que não há hábitos quanto à atividade física, que as horas destinadas à prática de atividade física nas escolas são insuficientes e que o rendimento escolar nas instituições de nível básico apresenta atrasos educacionais que devem ser enfrentados de forma abrangente.

Palavras-chave: atividade física, nível socioeconômico, desempenho acadêmico.

Fecha Recepción: Marzo 2021

Fecha Aceptación: Septiembre 2021

\section{Introducción}

Diversos estudios llevados a cabo en los últimos años han comprobado y demostrado los distintos beneficios que tiene la práctica regular de actividad física sobre el rendimiento escolar en estudiantes de diferentes niveles (Luque et al., 2021). Sin duda es un tema que no ha pasado desapercibido para los investigadores educativos. Bernal (2015) refiere el estudio de Shephard et al. (1984) como uno de los más significativos en el campo de la actividad física y el rendimiento escolar. Shephard et al. (1984) seleccionaron una escuela situada $25 \%$ por debajo del rendimiento escolar comparado 


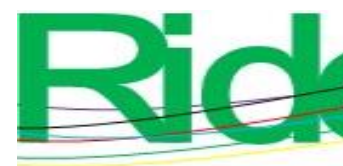

Revista Iberoamericana para la Investigación y el Desarrollo Educativo ISSN 2007 - 7467

con la media de otros colegios e implementaron un programa de actividades físicas a largo plazo; luego de cinco años el rendimiento escolar de dicha escuela incrementó $20 \%$.

La práctica recurrente de actividad física, además de los ya conocidos beneficios físicos que trae consigo, los cuales se ven reflejados en un estado óptimo de salud, también está asociada a la estimulación del aspecto mental, el aprendizaje, la concentración y el rendimiento académico de los escolares (Viteri y López, 2017). En efecto, la consulta de referentes empíricos demuestra que la actividad física en espacios educativos contribuye al desarrollo y mantenimiento de las funciones cognitivas de los alumnos, lo que se refleja en un mejor rendimiento académico (Rodríguez et al., 2020).

En México, sin embargo, a pesar de los beneficios ya nombrados, dentro de las instituciones educativas se mantienen niveles mínimos de ejercitación física con sesiones de 30-50 minutos una vez por semana, de acuerdo con los planes y programas de la Secretaría de Educación Pública [SEP] (2017). Aún más, Flores et al. (2017) encontraron que en términos reales el tiempo efectivo de una clase de 50 minutos de educación física se reduce a nueve minutos de actividades moderadas; el resto de la sesión se diluye en indicaciones, demostraciones y la organización propia de la clase.

Lo anterior revela la necesidad inmediata de investigaciones en México que prueben e impulsen programas integrales de actividades físicas escolares. Particularmente, en el estado de Querétaro existe el referente de Gómez, Méndez, Salazar y Cerezo (2012), quizá el único con esta temática, que incluyó una intervención a través de un programa interdisciplinario en una escuela primaria con el fin de combatir el sobrepeso y la obesidad durante el ciclo escolar 2012-2013. Al término, a pesar de que no era su objetivo central, el estudio demostró que el programa ayudó a mejorar de manera significativa el aprovechamiento escolar.

En virtud de lo anterior, se decidió llevar a cabo el presente estudio, el cual se estima cuantitativo, cuenta con un diseño cuasiexperimental de pretest, postest y grupo control y es de alcance correlacional a través de un muestreo no probabilístico.

Llegados a este punto, y antes de pasar a la siguiente sección, vale la pena señalar que aquí se entiende a la actividad física como cualquier movimiento producido por los músculos esqueléticos que demanda un gasto energético (Organización Mundial de la Salud [OMS], 2021), al ejercicio como toda aquella actividad planeada y estructurada (Escalante, 2011) y al deporte como aquella actividad privada que promueve el desarrollo de las competencias (Britapaz y Del Valle, 2015). 


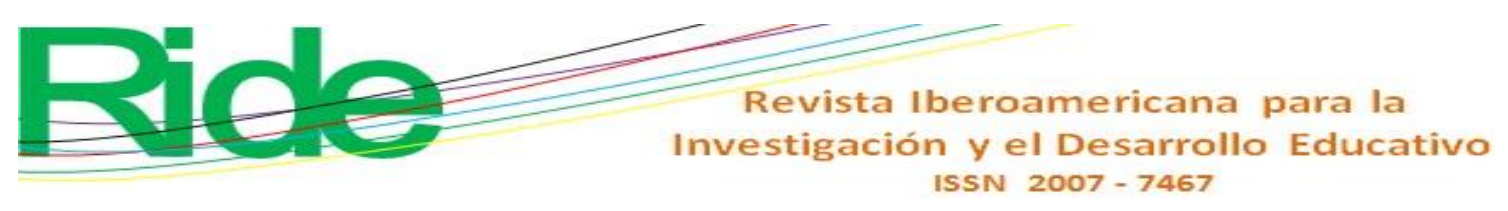

\section{Justificación}

A nivel mundial, el bajo rendimiento escolar ha comenzado a incrementarse en comparación con los años anteriores, así lo demuestra la Organización para la Cooperación y el Desarrollo Económico [OCDE] (9 de febrero de 2016) a través de su Programa Internacional para la Evaluación de los Estudiantes (PISA, por sus siglas en inglés), ya que 13 millones de estudiantes de los 65 países incorporados a la OCDE presentan bajo rendimiento escolar.

En 2012, $55 \%$ de los estudiantes de México tuvo un bajo rendimiento en matemáticas (media OCDE: $23 \%$ ), $41 \%$ en lectura (media OCDE: $18 \%$ ), $47 \%$ en ciencias (media OCDE: $18 \%$ ), y $31 \%$ en las tres materias (media OCDE: $12 \%$ ) (OCDE, 9 de febrero de 2016, p. 2).

De acuerdo con Nunes, Neves, Teodósio, Floriano y Lara (2014), la falta de actividad física en el nivel de educación primaria es un elemento que repercute en el retraso del desarrollo motor, lo que suele estar asociado con dificultades para el aprendizaje escolar. Para Manzano (2006), es indudable que la práctica de actividad física en el entorno escolar tiene beneficios cognitivos.

La presente investigación se realiza debido al conocimiento deficiente que registró una escuela pública de tiempo completo en la prueba Plan Nacional para la Evaluación de los Aprendizajes (Planea) del 2018 en las materias de Matemáticas y Español, principalmente. Otra de las razones por las cuales se realiza la investigación es la poca importancia que se le otorga a la actividad física en el plan curricular de educación básica y en los demás niveles educativos.

Por lo antes mencionado, el objetivo de la investigación es determinar la eficacia que tiene un programa de actividad física sobre el rendimiento escolar de estudiantes de una escuela primaria pública del municipio de Querétaro. Para dar respuesta a lo anterior, las hipótesis de trabajo buscan determinar la asociación del rendimiento escolar con la actividad y el nivel socioeconómico.

\section{Metodología}

Esta investigación es de tipo cuantitativa; cuenta con un diseño cuasiexperimental de pretest, postest y grupo control, y es de alcance descriptivo y correlacional. Se realizó una intervención durante el ciclo escolar 2018-2019 que incrementó las sesiones de la asignatura Educación Física de 60 a 300 minutos semanales en un grupo experimental, mientras que en un grupo control se mantuvo casi sin incremento. 
En lo referente al proceso de recolección de datos, inicialmente se solicitaron los permisos a las autoridades correspondientes. Posteriormente, se realizó el llenado de los consentimientos y asentimientos informados para la aplicación de los instrumentos. Después, se llevó a cabo una prueba piloto durante dos meses que validó los procesos e instrumentos utilizados. Finalmente, se efectuó la intervención con una duración de nueve meses.

\section{Muestra}

El estudio contempló un muestreo no probabilístico de tipo homogéneo por conveniencia. Los grupos fueron elegidos durante la transición que ocurre entre el preescolar y el primer año de primaria. Se seleccionaron los dos grupos de primer grado de una escuela pública urbana de tiempo completo con un total de 73 estudiantes. En la tabla 1 se observa la distribución de la muestra por edad, sexo y grupo de investigación.

Tabla 1. Distribución de la muestra

\begin{tabular}{|c|c|c|}
\hline Variable & Frecuencia & Porcentaje \\
\hline \multicolumn{3}{|c|}{ Edad } \\
\hline 5 años & 28 & $38.4 \%$ \\
\hline 6 años & 45 & $61.6 \%$ \\
\hline \multicolumn{3}{|c|}{ Sexo } \\
\hline Mujer & 35 & $47.9 \%$ \\
\hline Hombre & 38 & $51.1 \%$ \\
\hline Experimental & Grupo & $41.1 \%$ \\
\hline Control & 30 \\
\hline
\end{tabular}

Fuente: Elaboración propia

\section{Instrumentos}

Para medir el tiempo, tipo y frecuencia de la actividad física se diseñó, validó y aplicó un instrumento que consta de 20 ítems con una escala de Likert de cinco opciones de respuesta y un coeficiente de fiabilidad de alfa de Cronbach de 0.796. Además, para obtener el rendimiento académico de los estudiantes, se utilizó el "Test de evaluación del rendimiento académico" (TERA), a través de 24 indicadores correspondientes a los campos del lenguaje y matemáticas (González, Delgado, Martín y Barba, 2004). 
Mientras que en la figura 2 se puede observar un incremento de estudiantes que presentaron coordinación normal: el grupo control hasta $3.33 \%$ y el grupo experimental $30.24 \%$. Si bien en ambos grupos se disminuyó la incidencia de aquellos que presentaron insuficiencia de la coordinación, en el grupo experimental el cambio fue más pronunciado.

Figura 2. Postest de la batería de coordinación motriz global (KTK)

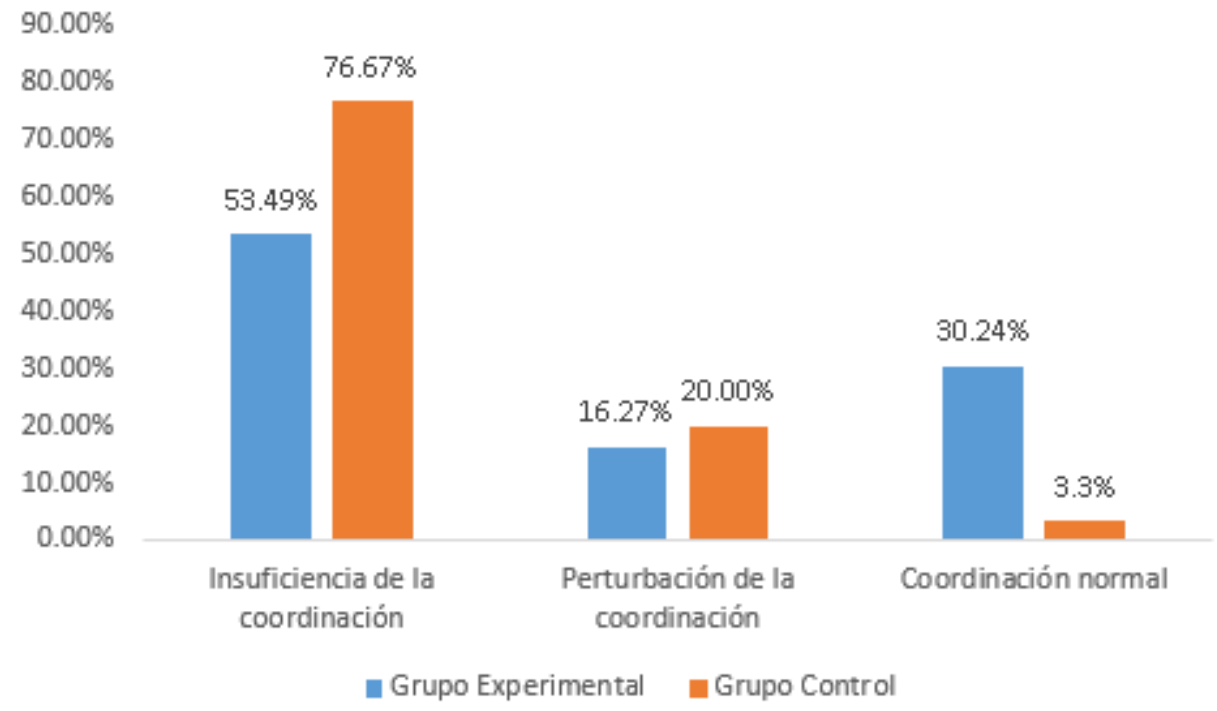

Fuente: Elaboración propia

Por su parte, la figura 3 exhibe los resultados del pretest del TERA. En el grupo control más de $55 \%$ presentó un conocimiento deficiente y solo $43.33 \%$ un conocimiento bueno, mientras que en el grupo experimental solamente $18.60 \%$ fue considerado apto, de acuerdo con el cumplimiento de ciertos ítems.

Figura 3. Pretest del rendimiento escolar TERA

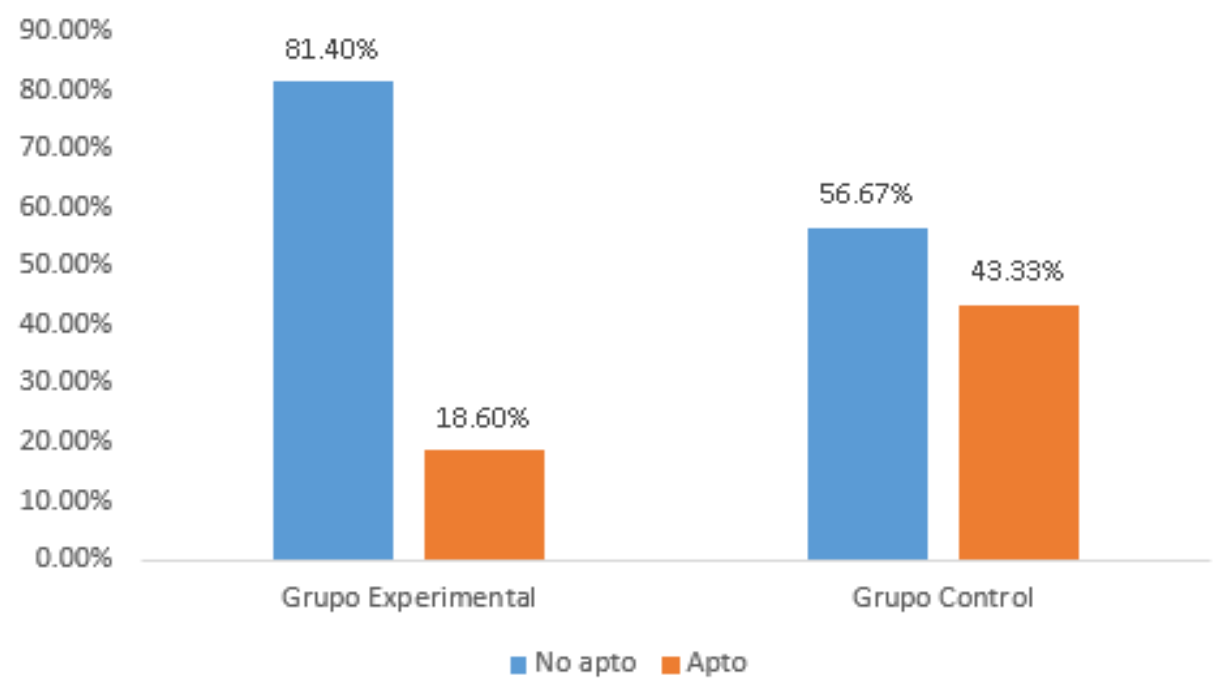

Fuente: Elaboración propia 


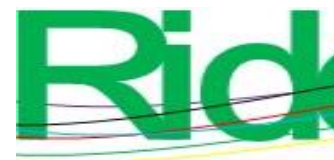

Revista Iberoamericana para la Investigación y el Desarrollo Educativo ISSN $2007-7467$

Más adelante, al contar con los resultados del postest de la aplicación del TERA, se pudo identificar que ambos grupos presentaron una mejoría en su rendimiento escolar. El grupo control fue el que mostró mayor incidencia $(63.33 \%)$, aunque el grupo experimental registró una cifra apenas menor (51.16\%) (ver figura 4).

Figura 4. Postest del rendimiento escolar TERA

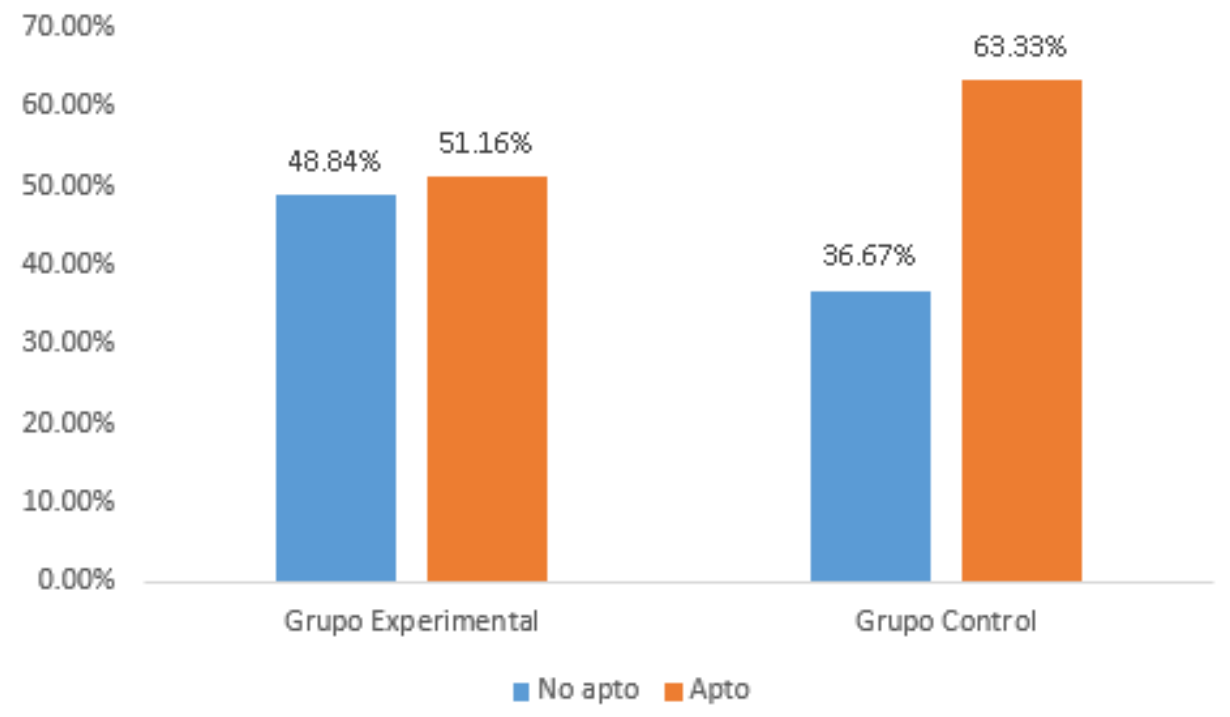

Fuente: Elaboración propia

Ahora bien, en la tabla 2 se puede observar el impacto que tuvo el haber incrementado a una hora diaria la actividad física, en lugar de una sola hora semanal, sobre el desarrollo de la coordinación motriz global, con un 0.001 de grado de significancia.

Tabla 2. Asociación de la actividad física con la coordinación motriz global del grupo experimental

\begin{tabular}{|c|c|c|c|c|c|c|c|c|}
\hline \multirow[t]{2}{*}{ Variables } & \multicolumn{5}{|c|}{ Diferencias relacionadas } & \multirow[b]{2}{*}{$\mathrm{T}$} & \multirow[b]{2}{*}{ Gl } & \multirow[b]{2}{*}{$\begin{array}{c}\text { Sig. } \\
\text { (bilatera } \\
\text { 1) }\end{array}$} \\
\hline & Media & $\begin{array}{l}\text { Desv. } \\
\text { típica }\end{array}$ & $\begin{array}{l}\text { Error } \\
\text { típico de } \\
\text { la media }\end{array}$ & Inferior & Superior & & & \\
\hline $\begin{array}{l}\text { Coordinación } \\
\text { global (pre) }\end{array}$ & -0.41860 & 0.73136 & 0.11153 & -0.64368 & -0.19353 & -0.3 .753 & 42 & $0.001 * *$ \\
\hline $\begin{array}{l}\text { Coordinación } \\
\text { global (pos) }\end{array}$ & & & & & & & & \\
\hline
\end{tabular}

Fuente: Elaboración propia 
Mientras que en la tabla 3 se muestra el grado de significancia obtenido en el grupo control al añadir una hora más de actividad física semanal además de la de su clase de Educación Física. Si bien es cierto que existieron mejoras, no tuvo un impacto significativo.

Tabla 3.Asociación de actividad física con la coordinación motriz global del grupo control

\begin{tabular}{|c|c|c|c|c|c|c|c|c|}
\hline \multirow[t]{2}{*}{ Variables } & \multicolumn{5}{|c|}{ Diferencias relacionadas } & \multirow[b]{2}{*}{$\mathrm{T}$} & \multirow[b]{2}{*}{ gl } & \multirow[b]{2}{*}{$\begin{array}{c}\text { Sig. } \\
\text { (bilateral) }\end{array}$} \\
\hline & Media & $\begin{array}{l}\text { Desv. } \\
\text { típica }\end{array}$ & $\begin{array}{l}\text { Error } \\
\text { típico de } \\
\text { la media }\end{array}$ & Inferior & Superior & & & \\
\hline $\begin{array}{c}\text { Coordinación } \\
\text { global } \\
\text { (pretest) } \\
\text { Coordinación } \\
\text { global } \\
\text { (postest) }\end{array}$ & -0.1333 & 0.50742 & 0.09264 & -0.32281 & -05614 & -1.439 & 29 & 0.161 \\
\hline
\end{tabular}

Fuente: Elaboración propia

Ahora bien, en la tabla 4 se puede observar la relación que existe entre la actividad física y el rendimiento escolar. Mediante la técnica $t$ de Student se obtuvo 0.000. Esto es: haber incrementado el tiempo y días de actividad física en el grupo experimental resultó altamente significativo.

Tabla 4. Asociación de actividad física con el rendimiento escolar del grupo experimental

\begin{tabular}{|c|c|c|c|c|c|c|c|c|}
\hline \multirow[t]{2}{*}{ Variables } & \multicolumn{5}{|c|}{ Diferencias relacionadas } & \multirow[b]{2}{*}{$\mathrm{T}$} & \multirow[b]{2}{*}{$\mathrm{gl}$} & \multirow[b]{2}{*}{$\begin{array}{c}\text { Sig. } \\
\text { (bilateral } \\
\text { ) }\end{array}$} \\
\hline & Medía & $\begin{array}{l}\text { Desv. } \\
\text { típica }\end{array}$ & $\begin{array}{l}\text { Error } \\
\text { típico de } \\
\text { la media }\end{array}$ & Inferior & Superior & & & \\
\hline $\begin{array}{l}\text { Rendimiento } \\
\text { escolar (pre) }\end{array}$ & 0.32558 & 0.47414 & 0.07231 & 0.17966 & -47150 & 4.503 & 42 & $0.000 * * *$ \\
\hline $\begin{array}{l}\text { Rendimiento } \\
\text { escolar (pos) }\end{array}$ & & & & & & & & \\
\hline
\end{tabular}

Fuente: Elaboración propia 
Y en la tabla 5 se muestra que en el grupo control también existió significancia, debido a la hora extra semanal, pero no fue tan significativa como la que se obtuvo en el grupo experimental.

Tabla 5. Asociación de actividad física con el rendimiento escolar del grupo control

\begin{tabular}{|c|c|c|c|c|c|c|c|c|}
\hline \multirow[t]{2}{*}{ Variables } & \multicolumn{5}{|c|}{ Diferencias relacionadas } & \multirow[b]{2}{*}{$\mathrm{T}$} & \multirow[b]{2}{*}{$\mathrm{gl}$} & \multirow[b]{2}{*}{$\begin{array}{c}\text { Sig. } \\
\text { (bilateral } \\
\text { ) }\end{array}$} \\
\hline & Medía & $\begin{array}{l}\text { Desv. } \\
\text { típica }\end{array}$ & $\begin{array}{l}\text { Error } \\
\text { típico de } \\
\text { la media }\end{array}$ & Inferior & Superior & & & \\
\hline $\begin{array}{l}\text { Rendimiento } \\
\text { escolar (pre) } \\
\text { Rendimiento } \\
\text { escolar (pos) }\end{array}$ & 0.23333 & 0.43018 & 0.07854 & 0.07270 & 0.39397 & 2.971 & 29 & $0.006 * *$ \\
\hline
\end{tabular}

Fuente: Elaboración propia

Finalmente, en la tabla 6 se observa la relación entre el nivel socioeconómico del estudiante y otras variables. Aquí resultó altamente significativa la interacción con las variables relacionadas con el nivel de estudios del padre o madre de familia y el medio de transporte que usa el estudiante para llegar a la escuela.

Tabla 6. Relación del nivel socioeconómico con las variables TERA, KTK y actividad física utilizando la prueba de ji al cuadrado

\begin{tabular}{|c|c|c|}
\hline Variables & $\chi_{\text {cal }}^{2}$ & Pvalue \\
\hline Actividad física (frecuencia) & 8.746 & 0.890 \\
\hline Actividad física (tiempo) & 17.211 & 0.639 \\
\hline Nivel de estudios & 98.956 & $0.000 * * *$ \\
\hline Pretest TERA & 9.756 & 0.082 \\
\hline Pretest KTK & 12.919 & 0.228 \\
\hline Postest TERA & 6.655 & 0.248 \\
\hline Postest KTK & 5.156 & 0.881 \\
\hline Medio de transporte & 24.993 & $0.005 * *$ \\
\hline ***Altamente significativo **Muy significativo *Significativo & \\
\hline
\end{tabular}

Fuente: Elaboración propia 


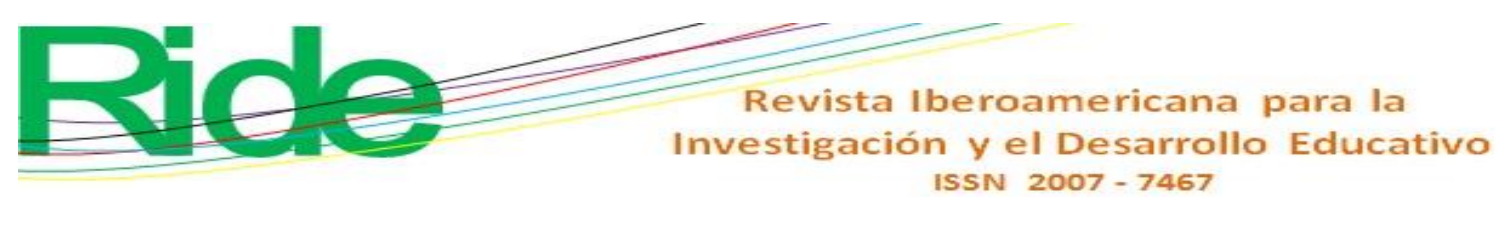

\section{Discusión}

El objetivo del estudio se centró en analizar el impacto que tendría incrementar el volumen de actividad física en estudiantes de nivel primaria que obtuvieron calificaciones insuficientes de rendimiento escolar en la prueba Planea 2018.

En lo que refiere al rendimiento académico, la OCDE (9 de febrero de 2016) reportó que en los últimos años el bajo rendimiento escolar se ha incrementado hasta $11 \%$, sobre todo en las áreas de matemática, ciencia y lenguaje. Aquí los resultados arrojados por el pretest de TERA revelaron que en el grupo de intervención $81.4 \%$ de los estudiantes obtuvieron niveles deficientes (no aptos) del conocimiento en los rubros de español y matemáticas. No obstante, el panorama cambió con la aplicación del programa de actividad física cuya duración fue de nueve meses, ya que los datos obtenidos por el postest describen una disminución en la cantidad de estudiantes con condición deficiente, al situarse en $48.84 \%$, lo que respalda la asociación positiva que tiene la práctica de la actividad física regular sobre el rendimiento escolar en estudiantes de nivel primaria.

Asimismo, en lo que respecta a la actividad física, la OMS (26 de noviembre de 2020), dentro de sus recomendaciones mundiales sobre la actividad física para la salud, señala que niños y adolescentes entre 5-17 años deben realizar mínimamente 60 minutos diarios de actividad moderada o vigorosa. Sin embargo, los datos del estudio arrojaron que en el grupo experimental $58.13 \%$ de los estudiantes no cumplen con las cantidades mínimas de actividad física, situación que, además de representar un factor para el desarrollo de enfermedades como el sobrepeso y la obesidad (OMS, 26 de noviembre de 2020), también constituye un obstáculo importante para el rendimiento académico.

En virtud de lo anterior, el programa de intervención logró incrementar el volumen de actividad física de 60 a 300 minutos semanales, y trajo consigo modificaciones tangibles y sustanciales para los estudiantes tanto en el incremento del rendimiento escolar como en la mejora de capacidades físicas coordinativas. Los resultados obtenidos se suman a los referentes empíricos que han demostrado que una mayor cantidad de actividad física se asocia con mejores indicadores de rendimiento escolar, como lo demostraron Trullén (2020) en estudiantes españoles de primaria y Quílez (2020) en alumnos de nivel secundaria. También, los resultados reflejan consistencia con el incremento de las capacidades coordinativas a través de la actividad física, tal y como lo enfatiza el estudio de Benálcazar (2020) en niños ecuatorianos. Además, al igual que en la investigación de Urquidez et al. (2017), la intervención con actividad física brindó mejoras en aspectos educativos y de salud. 


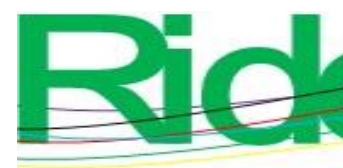

Revista Iberoamericana para la Investigación y el Desarrollo Educativo ISSN 2007 - 7467

Todavía más: los hallazgos encontrados comparten similitud con estudios de intervención efectuados en otros países, como el realizado en Suecia por Fritz, Cöster, Rosengren, Karlsson y Karlsson (2020), quienes, al igual que en el presente estudio, incrementaron la actividad física en niños de 6-8 años de 60 a 200 minutos semanales, aunque ellos lo hicieron durante un periodo de nueve años. A pesar de la diferencia de tiempo de implementación, en ambos se encontró asociación entre el aumento de la actividad física y el incremento del rendimiento escolar (aunque respecto a este último indicador, se midió de forma distinta, allá mediante las calificaciones escolares [Suecia] y acá por medio de una prueba estandarizada [México]). Además, aunque ambas investigaciones consideraron la variable sexo, solo el estudio de Fritz et al. (2020) encontró asociación significativa al reconocer que las niñas presentaban mejores niveles de rendimiento escolar.

Por otra parte, es importante reconocer que a pesar de haber obtenido resultados contundentes sobre el impacto que tiene la actividad física en el rendimiento escolar y las habilidades físicas coordinativas de los estudiantes, la investigación no arrojó datos significativos sobre la relación entre variables como el nivel socioeconómico o el sexo, aunque es preciso resaltar que la intervención se realizó en una escuela de nivel económico medio típico-alto. Lo anterior sugiere la posibilidad de ampliar el alcance del programa de intervención hacia escuelas de diversas localidades y distintos niveles socioeconómicos.

\section{Conclusiones}

Con base en los resultados del estudio se rechaza la hipótesis de trabajo $\mathrm{H}_{1}$, ya que el nivel socioeconómico de la familia no influye sobre el rendimiento académico de los estudiantes y también se descarta la hipótesis de trabajo $\mathrm{H}_{2}$, ya que los niños que tienen un bajo nivel socioeconómico no tuvieron mejor desarrollo motriz. Por el contrario, se acepta que el desarrollo motor de los niños mejora con la práctica regular de actividad física. E igualmente que algunas variables de la actividad física (duración, frecuencia, intensidad, hidratación, entre otros) se asociaron con la disminución de peso corporal o modificaciones en la composición corporal.

También se encontró una correlación significativa entre el tiempo y la frecuencia que se emplea para realizar actividades físico deportivas y el rendimiento académico. De igual manera, se observó una asociación altamente significativa entre los niños que realizan actividades físico deportivas diariamente y el rendimiento escolar. Además, se registró una asociación altamente significativa en el rendimiento escolar deficiente y la 


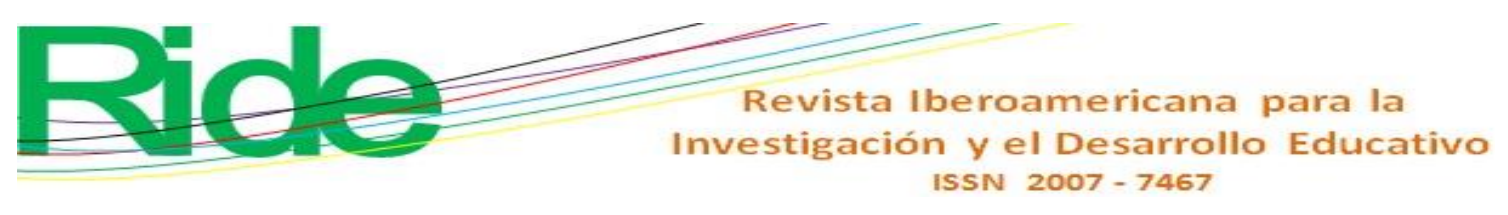

insuficiencia de la coordinación. Asimismo, se apreció una vinculación entre el rendimiento escolar insuficiente y las variables de la actividad física (ningún deporte practicado, días a la semana, tiempo entre semana y tiempo en los fines de semana). Y se detectó una asociación altamente significativa entre el rendimiento escolar deficiente y los días que observan televisión y tiempo que les dedican a los videojuegos (tiempo que le dedican entre semana, tiempo que le dedican los fines de semana, días entre semana que le dedican y días que le dedican los fines de semana).

Aunado a todo lo anterior, se detectó una asociación altamente significativa entre el rendimiento escolar deficiente y la insuficiencia de la coordinación global en casi toda la muestra y una asociación altamente significativa entre el programa de actividad física y la mejora del desarrollo motor.

Un aporte significativo del presente estudio radica en señalar que el proceso cognitivo que emprendieron los estudiantes en la presente investigación (seis a siete años) estuvo vinculado con el déficit de la coordinación global, el nivel de atenciónconcentración, el sedentarismo y el tono muscular, entre otros factores. Por lo que realizar un esquema de actividades físico deportivas beneficia a la población a salir del sedentarismo, a mejorar la coordinación global, a mejorar los procesos de mielinización para una mejor conexión sináptica, con en el aumento de la oxigenación en las células, lo que provoca un aumento en la atención-concentración y produce autonomía, autoconfianza y reconocimiento de la corporeidad, fundamentales para una buena coordinación global.

Cabe resaltar que el tipo de escuela de tiempo completo se asoció de manera altamente significativa con niveles muy bajos de actividad física y la poca realización del deporte y actividad física extraescolar. Las personas que resultaron no tener un rendimiento académico apto según la prueba del TERA, después de concluir con el programa de actividad física, pasaron a tenerlo según la misma prueba. Por lo tanto, se logró la vinculación del programa de actividad física con las materias Español y Matemáticas. Con intervenciones de este tipo se mantienen activos los estudiantes, reforzamos temas del salón de clase, los estudiantes se divierten y aprenden de una forma kinestésica, todo lo cual da resultado ser más capaces académicamente.

Por otro lado, el estudio identificó una asociación entre el rendimiento escolar y el tiempo dedicado a la actividad física o deporte, con quién vive el estudiante, nivel de estudios de los padres, tiempo dedicado a ver televisión y tiempo dedicado a los videojuegos. Al tener conocimiento de estos factores asociados, se enfatiza la importancia de realizar actividad física, por lo menos cuatro días a la semana con 30 minutos por día, 
con una alimentación balanceada y buena práctica de hidratación, tener apoyo de los familiares o tutores para fortalecer deseos de continuar realizando la actividad física y consolidar valores y hábitos, mantener conversaciones con los padres o tutores, así como con los compañeros, de su actividad física para mantener o mejorar la autoconfianza, la autonomía, la toma de decisiones y la atención-concentración, actitudes que serán benéficas para seguir mejorando el rendimiento escolar y reducir el tiempo de televisión y videojuegos.

Finalmente, es importante recalcar que el rendimiento escolar insuficiente o no apto es un fenómeno que se asocia con los hábitos de casa y el tiempo de actividad física. Es un problema para el desarrollo de la comunidad que requiere prevención para evitar riesgos como el sobrepeso, la obesidad, problemas psicológicos, enfermedades crónicas, entre otras cosas, sin dejar de lado el estancamiento y la poca utilidad en la vida cotidiana que tendrán los escolares por el deficiente rendimiento escolar.

\section{Futuras líneas de investigación}

Por último, para próximas investigaciones se estiman como áreas de oportunidad interesantes el terreno de las habilidades socioemocionales, los valores y los estilos de vida saludables, ya que se observaron resultados implícitos en la mejora de la interacción de los niños, el incremento de la atención y la concentración, la presencia de valores como el respeto, la tolerancia, la honestidad, la empatía y la capacidad para dialogar con sus compañeros. También, se detectaron hábitos como llevar botella de agua natural para la clase de Educación Física, procurar una fruta para consumir al término de la clase, el aseo corporal con frecuencia, disminución del ausentismo en clases y reducción del tiempo semanal dedicado a la televisión y a los videojuegos.

En virtud de lo anterior, se plantean líneas de investigación emergentes en el ámbito de educación física para la salud y el bienestar integral, en donde se consideren variables como el estado nutricional, los hábitos alimenticios, el autoconcepto físico y el uso del tiempo libre. Asimismo, se sugiere que un mayor tiempo de intervención y una población más amplia permitirán correlaciones interesantes y significativas para próximos estudios. 


\section{Referencias}

Asociación Mexicana de Agencias de Inteligencia de Mercado y Opinión [AMAI]. (2017). Nivel Socio Económico AMAI 2018. Recuperado de http://www.cua.uam.mx/pdfs/coplavi/s_p/doc_ng/metodologia-nse-2018amai.pdf.

Bernal, D. (2015). Educación física: una asignatura para mejorar el rendimiento académico, la cognición y los valores. (Trabajo de fin de grado de maestría). Universidad de Salamanca, Salamanca. Recuperado de https://gredos.usal.es/jspui/handle/10366/125965.

Benálcazar, Y. (2020). Relación de la coordinación motora y el rendimiento académico en niños de 6 a 8 años que asisten a la unidad educativa Jorge Peñaherrera del cantón Pimampiro. (Tesis de licenciatura). Universidad Técnica del Norte, Ibarra. Recuperado de http://repositorio.utn.edu.ec/handle/123456789/10194.

Britapaz, L. y Del Valle, J. (2015). Significado del deporte en la dimensión social de la salud. Salus, 19, 27-33.

Escalante, Y. (2011). Actividad física, ejercicio físico y condición física en el ámbito de la salud pública. Revista Española de Salud Pública, 85(4), 325-328.

Flores, P. J., Salazar, C., Gómez, J. A., Barreto, Y., Valdovinos, O., Vicente, J. U. y Del Río, J. E. (2017). Medición del tiempo efectivo de la clase de educación física y su impacto en el gasto calórico en escolares de nivel primaria del municipio de Colima, México. Sportis. Scientific Journal of School Sport, Physical Education and Psychomotricity, 3(1), 34-49. Recuperado de http://dx.doi.org/10.17979/sportis.2017.3.1.1766.

Fritz, J., Cöster, M., Rosengren, B., Karlsson, C. and Karlsson, M. (2020). Daily School Physical Activity Improves Academic Performance. Sports, 8(6). Retrieved from https://www.mdpi.com/2075-4663/8/6/83.

Gómez, O. A., Méndez, J. C., Salazar, M. E. y Cerezo, F. (2012). Programa interdisciplinario de intervención para la prevención de la obesidad infantil. Universidad Autónoma de San Luis Potosí. Recuperado de https://onedrive.live.com/?authkey=\%21AAwSe8g3ZJGyL8I\&cid=BECB78822 F793C

0D\&id=BECB78822F793C0D\%211607\&parId=BECB78822F793C0D $\% 21354$ $\& o=$ On eUp. 
González, M. J., Delgado, M. J., Martín, I. y Barba, M. J. (2004). Test de evaluación del rendimiento académico en educación infantil y primaria (TERA). (Trabajo no publicado). Universidad de Málaga, Málaga.

Kiphard, E, J. and Schilling, F. (1974). Körperkoordinationstest für Kinder. Germany: Weinheim.

Luque, A., Gálvez, A., Gómez, L., Escámez, J., Tárraga, L. y Tárraga, P. (2021). ¿Mejora la actividad física el rendimiento académico en escolares? Una revisión bibliográfica. Journal of Negative \& No Positive Results, 6(1), 84-103. Recuperado de https://revistas.proeditio.com/jonnpr/article/view/3277.

Manzano, J. (2006). Educación física y desarrollo integral. Isla de Arriarán, (28), 275294.

Nunes, L. de C., Neves, D., Teodósio, G. de F., Floriano, P. M. e Lara, S. (2014). Perfil de estudantes dos anos iniciais com baixo rendimento escolar: importância da educação física na escola. Reviste Brasilera de Ciência e Movimento, 22(2), 3646.

Organización Mundial de la Salud [OMS]. (2004). Estrategia mundial sobre régimen alimentario, actividad física y salud. Ginebra, Suiza: Organización Mundial de la Salud.

Organización Mundial de la Salud [OMS]. (26 de noviembre de 2020). Actividad física. Recuperado de https://www.who.int/es/news-room/fact-sheets/detail/physicalactivity.

Organización para la Cooperación y el Desarrollo Económicos [OCDE]. (9 de febrero de 2016). Estudiantes de bajo rendimiento: Por qué se quedan atrás y cómo ayudarles a tener éxito. Resumen México. Recuperado de https://www.oecd.org/centrodemexico/medios/PISA\%20Low\%20Performing\%2 0Students\%20Press\%20Handout\%20MEXICO\%20FINAL.pdf.

Quílez, M. (2020). Relación entre la actividad física y el rendimiento académico. Análisis y comparación por sexo y edad. Campus Educación. Revista Digital Docente, 5(17). 32-37. Recuperado de https://www.campuseducacion.com/revista-digitaldocente/numeros/17.

Rodríguez, A., Rodríguez, J., Guerrero, H., Arias, E., Paredes, A. y Chávez, V. (2020). Beneficios de la actividad física para niños y adolescentes en el contexto escolar. Revista Cubana de Medicina General Integral, 36(2). Recuperado de http://scielo.sld.cu/pdf/mgi/v36n2/1561-3038-mgi-36-02-e1535.pdf. 


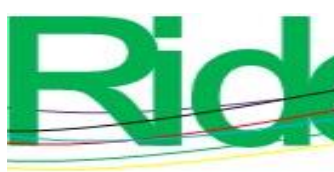

Secretaría de Educación Pública [SEP]. (2017). Aprendizajes clave para la educación integral. Educación Física. Educación básica. Plan y programas de estudio, orientaciones didácticas y sugerencias de evaluación. Ciudad de México, México: Secretaría de Educación Pública. Recuperado de https://www.planyprogramasdestudio.sep.gob.mx/descargables/biblioteca/basica -educ-fisica/1LpMEducacion-Fisica_Digital.pdf.

Secretaría de Educación Pública [SEP]. (2018). Plan Nacional para la Educación de los Aprendizajes. Informe de resultados de mi escuela. Recuperado de http://143.137.111.129/PLANEA/planea_re_18_basica/content/pages/basica/esc uela.php.

Shephard, R., Volle, M., Lavallée, H., LaBarre, R., Jéquier, J. and Rajic, M. (1984). Required physical activity and academic grades: A controlled study. In Hmarinen, J. and Välimäki, I. (eds.), Children and Sport (pp. 58-63). Verlag, Germany: Springer.

Trullén, C. (2020). Relación entre factores sociodemográficos, actividad física extraescolar y rendimiento académico en estudiantes de educación primaria. Revista Digital de Educación Física, 11(63). 60-79. Recuperado de http://emasf.webcindario.com/Relacion_entre_factores_sociodemograficos_activ idad_fisica_extraescolar_y_rendimiento_academico.pdf.

Urquidez, R., Ramírez, D., Ramos, A., Rodriguez, A., Wall, A., Díaz, B. A., Medrano, G., Quizán, T. y Esparza, J. (2017). Promoción de la actividad física en niños del norte de México: efectividad de una intervención educativa. Acta Universitaria, 27(2), 32-38.

Viteri, M. F. y López, I. A. (2017). La actividad física en el rendimiento académico de los niños de quinto, sexto y séptimo año en la Escuela de Educación Básica Gregoire Girard. (Proyecto de investigación). Universidad Técnica de Ambato, Ambato. Recuperado de http://repositorio.uta.edu.ec/jspui/handle/123456789/25172. 


\begin{tabular}{|c|c|}
\hline Rol de Contribución & Autor (es) \\
\hline Conceptualización & Francisco De Jesús Ávila Manríquez \\
\hline Metodología & $\begin{array}{l}\text { Julio César Méndez Ávila (Principal) } \\
\text { José Miguel Silva Llaca (Igual) }\end{array}$ \\
\hline Software & $\begin{array}{l}\text { Programación, desarrollo de software; Diseño de programas } \\
\text { informáticos; Implementación del código informático y } \\
\text { algoritmos de soporte; Pruebas de componentes de código } \\
\text { existentes. }\end{array}$ \\
\hline Validación & $\begin{array}{l}\text { Francisco De Jesús Ávila Manríquez (Principal) } \\
\text { Julio César Méndez Ávila (Igual) } \\
\text { José Miguel Silva Llaca (Ayuda) }\end{array}$ \\
\hline Análisis Formal & Julio César Méndez Ávila \\
\hline Investigación & $\begin{array}{l}\text { Francisco De Jesús Ávila Manríquez (Principal) } \\
\text { Julio César Méndez Ávila (Igual) } \\
\text { José Miguel Silva Llaca (Ayuda) }\end{array}$ \\
\hline Recursos & Oscar Ángel Gómez Terán \\
\hline Curación de datos & $\begin{array}{l}\text { Actividades de gestión (producir metadatos), depurar } \\
\text { información y mantener datos de investigación (incluyendo } \\
\text { código de software, donde sea necesario para interpretar los } \\
\text { datos en sí) para uso inicial y posterior reutilización. }\end{array}$ \\
\hline $\begin{array}{l}\text { Escritura - Preparación del } \\
\text { borrador original }\end{array}$ & $\begin{array}{l}\text { Francisco De Jesús Ávila Manríquez (Principal) } \\
\text { Julio César Méndez Ávila (Ayuda) }\end{array}$ \\
\hline Escritura - Revisión y edición & $\begin{array}{l}\text { Julio César Méndez Ávila (Principal) } \\
\text { José Miguel Silva Llaca (Ayuda) }\end{array}$ \\
\hline Visualización & Francisco De Jesús Ávila Manríquez \\
\hline Supervisión & $\begin{array}{l}\text { Francisco De Jesús Ávila Manríquez (Principal) } \\
\text { Julio César Méndez Ávila (igual) } \\
\text { José Miguel Silva Llaca (Ayuda) } \\
\text { Oscar Ângel Gómez Terán (Ayuda) }\end{array}$ \\
\hline Administración de Proyectos & $\begin{array}{l}\text { Francisco De Jesús Ávila Manríquez (Principal) } \\
\text { Julio César Méndez Ávila (Igual) }\end{array}$ \\
\hline Adquisición de fondos & $\begin{array}{l}\text { Oscar Ángel Gómez Terán (Principal) } \\
\text { Julio César Méndez Ávila (Ayuda) }\end{array}$ \\
\hline
\end{tabular}

